\title{
Distal demonstratives licensed by culturally-familiar scenarios
}

\author{
Ryan B. Doran \& Gregory Ward*
}

\begin{abstract}
Previous work on English demonstratives (Gundel et al. 1993, Diessel 1999) has shown that distal demonstratives may be used to indicate information status. In this paper, we investigate a use of distal demonstratives that is licensed by (presumed) shared knowledge of culturally-familiar scenarios, rather than by private shared knowledge of particular entities, as exemplified in (1):

(1) I'm not really fit to talk in the morning until I've had that first cup of coffee. [corpus] Here, the entity specified by the demonstrative NP stands proxy for the familiar routine of beginning's one day and no specific cup of coffee is being referred to. We show that, by using the demonstrative in this way, the speaker presumes that the hearer is a member of community in which the relevant scenario is familiar.
\end{abstract}

Keywords: Demonstratives; Pragmatics; Semantics

In this paper, we identify and analyze a use of the English distal demonstrative that has not been discussed in the previous literature on demonstratives, as shown in (1):

(1) a. Megan shared her story of the realities of a military wife, and how she felt the day she got that knock on the door. [corpus]

b. I'm not really fit to talk in the morning until I've had that first cup of coffee. [corpus]

c. That's how we've always moved this country forward. By all of us coming together on behalf of our children. Folks who volunteer to coach that team, to teach that Sunday School class. Because they know it takes a village.... [corpus]

In each of the examples in (1), the speaker is using the underlined demonstrative NP to refer to an entity whose assumed familiarity is based on the existence of a shared cultural scenario.

Our claim is that this use is licensed when the speaker has a basis for presuming familiarity with a culturally-salient scenario (Fillmore 1982), which can be specified directly by the demonstrative itself or can be evoked by an entity stereotypically associated with that scenario. This use thus shares certain features with other uses of the distal demonstrative that presume familiarity, but the basis for the familiarity that licenses this particular use is distinct. Specifically, we argue that the presumed familiarity that licenses this use is grounded in community co-presence (Clark \& Marshall 1981). That is, community members, in virtue of their membership, can be assumed to be familiar with the relevant scenario and, even in the absence of actual membership, community co-presence may be inferred. We will first review the previous literature on related uses of demonstratives and show that this use is distinct, and then turn to examine the basis for familiarity that licenses this use.

In the canonical use of the distal demonstrative, the speaker refers ostensively to a specific entity in the immediate spatio-temporal context (Kaplan 1989, Diessel 1999).

I spilled that cup of coffee. [pointing to a cup of coffee]

\footnotetext{
* Authors: Ryan B. Doran, University of Regina (ryan.doran@uregina.ca), \& Gregory Ward, Northwestern University (gw@northwestern.edu).
} 
Here, the intended referent is a particular cup of coffee in the immediate perceptual environment that is pointed out by the speaker's demonstration. Another related use of the distal is to refer to specific entities that are assumed to be known to the hearer on the basis of private shared knowledge (Joshi 1982, Gundel et al. 1993, Levinson 2004, inter alia), as in (3):

(3) That dog (next door) kept me awake. [=Gundel et al. 1993, ex. (5)]

Here, the speaker is referring to a particular dog, which is assumed to be already known to the hearer. The familiarity required for the felicity of the demonstrative in (3) is satisfied by the (presumed) shared knowledge that is specific to this speaker-hearer dyad, rather than general knowledge that any speaker can be assumed to share.

In our previous work (Doran \& Ward 2015a,b), we have identified a variety of uses that are licensed by presumed shared knowledge of the socio-cultural context, rather than by private shared knowledge of specific entities.

(4) a. Look, the dealers are the popular kids, but they're not normal popular. They're these crunchy granola dudes that have convinced everyone that they're cool. [=D\&W 2015a, \#1b]

b. The inspector himself was excellent... He's this short guy with very little hair and a pronounced working-class English accent. It was like having Phil Collins inspect your house. [=D\&W 2015a, \#20]

In these examples, the felicitous use of the demonstrative depends upon the speaker and hearer sharing relevant cultural information, rather than knowledge about specific entities. In (4a), the speaker is classifying the referents with respect to a culturally salient category, rather than referring to any specific entity, i.e. the dealers in question are being classified as crunchy granola dudes. Similarly, we find that socio-cultural knowledge can license a distributed interpretation of demonstratives, as in (5):

(5) a. Quicklimos Wedding Limousine Services. Every bride looks forward to this day. [=D\&W 2015b, \#7a]

b. Getting a puppy is exciting for everyone, but those first few weeks at home can shape the dog he will grow to be. [=D\&W 2015b, \#7b]

In, e.g. (5a), the demonstrative is interpreted distributively, rather than referentially. Here the demonstrative NP specifies the wedding day of each individual bride, which is licensed by the presumed shared knowledge that brides have associated with them a particular wedding day.

The use of the distal that we are concerned with in this paper displays features from each of the examples in (3)-(5): sensitivity to familiarity and to the socio-cultural context of utterance. Consider the examples in (1), repeated below:

(6) a. Megan shared her story of the realities of a military wife, and how she felt the day she got that knock on the door. [corpus]

b. I'm not really fit to talk in the morning until I've had that first cup of coffee. [corpus]

c. That's how we've always moved this country forward. By all of us coming together on behalf of our children. Folks who volunteer to coach that team, to teach that Sunday School class. Because they know it takes a village.... [corpus]

In each example, the relevant entities are identified via a presumed familiarity with socio-cultural stereotypes. In (6a), the speaker is assuming familiarity with the culturally-shared scenario in 
which a door knock stands proxy for the event of learning of a military family member's death. Likewise, in (6b), the speaker presumes that the hearer is familiar with the morning routine scenario involving the consumption of coffee; and in (6c) the speaker is assuming familiarity with scenarios involving volunteering for activities related to children.

This use of the distal demonstrative is licensed when the speaker has a basis for presuming familiarity with a culturally-familiar scenario, which the speaker evokes by means of the demonstrative NP. This use is thus distinct from the 'private shared knowledge' use of the distal in which reference is made to a specific discourse entity, as in (3) above. In support of this claim, we note first that in the absence of a relevant culturally-familiar scenario, infelicity results (on the intended interpretation). Consider (7):

a. Ready to buy that house/that car/that puppy? [corpus]

b. \#Ready to buy that vacuum cleaner/that garden hose/that sport coat?

In (7a-b), deictic and anaphoric interpretations are available. However, the demonstratives in (7a) also license an additional interpretation, given that there is a culturally-familiar scenario associated with buying these kinds of entities. For example, buying a house stereotypically involves house viewings, real estate agents, securing a mortgage, and so forth. This interpretation is not available for the demonstratives in (7b), as there is no appropriately familiar scenario associated with these kinds; there is, e.g., no routine that one typically goes through in order to purchase a vacuum. Thus, it is the availability of associated scenarios that licenses the demonstratives in (7a), but not in (7b).

Moreover, with this use we find that the demonstrative can either specify a culturallyfamiliar scenario directly or can specify an entity that stands proxy for an event or property within that scenario. Consider (8):

a. Why you absolutely need to take that vacation. [corpus]

b. New Car vs. Used Car. Advantages of buying new.... It has no history, no past, and it has that new car smell, too! [corpus]

In (8a), the vacation scenario is evoked directly through the use of the demonstrative NP and is licensed by the presumed familiarity of this scenario. In (8b), however, the relevant culturallyfamiliar scenario is that of buying a new car. Here, it is the smell of a new car - stereotypically representative of a new car - that the speaker is referring to with the demonstrative. The relevant scenario is specified by proxy, in which an element of the scenario stands in for the whole scenario.

When the scenario is evoked by a proxy relationship, the proxy relationship holds between the scenario and elements which are considered to be characteristic or emblematic of that scenario. Consider (9):

(9) a. Looking forward to that beer at the end of the day. [corpus]

b. \#Looking forward to that tea at the end of the day.

Here, the demonstrative in (9a) is felicitous given that a beer stands proxy for the 'relaxing-afterwork' scenario. This scenario is evoked by drinking beer, which is a characteristic element of that scenario. (Note that Looking forward to that glass of wine at the end of the day would also work in the same way). In (9b), however, while drinking tea can certainly be part of one's routine of relaxing after work, it is not stereotypically characteristic of that scenario. Thus, this demonstrative NP fails to evoke the relevant 'relaxing-after-work' scenario and its use is infelicitous without further information about the hearer's typical relaxing-after-work routine. 
What is essential for the felicity of the demonstrative on this interpretation is that the proxy element be stereotypically emblematic of the relevant scenario.

We propose that the relevant notion of familiarity that licenses this use of demonstratives is that of community membership (Clark \& Marshall 1981). That is, the speaker must have a plausible basis for believing that the hearer is a member of a certain social community and will thus be sufficiently familiar with the relevant scenario in virtue of their community membership. Consider (10):

(10) But most Americans have other things on their minds right now. Paying the bills. Finally taking that vacation. Baseball. They're not really paying a lot of attention to the campaign. [corpus]

Here, the writer is assuming that it is sufficiently familiar to a general American readership that taking a vacation is among the set of routine summer activities. Note that there is no one particular vacation being specified by the demonstrative; rather, taking a vacation is emblematic of typical summer activities for the relevant audience. In the context of this example, the writer seeks to evoke this particular scenario in making the point that the American electorate does not pay much attention to politics during the summer. Note further that the demonstrative could have also been felicitous in place of the definite in paying the bills. However, the use of the demonstrative with bills would have suggested a different scenario. Such a scenario would be typical or routine life activities in general, rather than typical summer activities, and thus would not be consistent with the writer's overall point in this passage.

Within a more specialized community, the presumed familiarity with the relevant scenario will be correspondingly limited to that community, as in (11).

Most hunters cherish that permit. [corpus]

For us, cultural scenarios involving hunting are not particularly familiar. But we nevertheless can infer that the speaker here is evoking a scenario in which, for the community of hunters, the permit stands proxy for the process of obtaining a hunting permit. Despite the fact that we are not co-present with the relevant community (in the sense of Clark \& Marshall 1981), we can see that the demonstrative here is felicitous because the speaker is assuming that there is a scenario associated with getting a hunting permit and that this scenario will be familiar to members of the hunting community.

Our account of this use also provides an explanation for the observation that a demonstrative NP may receive a distributed interpretation (King 2001, Elbourne 2008), as in (12):

(12) a. Most avid snow skiers remember that first black diamond run they attempted to ski. [=King $2001 \mathrm{Ch} 1$ \#5]

b. $\quad$ Every father dreads that moment when his oldest child leaves home. [=King 2001 Ch 1 \#4]

In these examples, the demonstrative NPs can receive an interpretation in which their value covaries with the preceding quantified NP. In (12a), for example, the relevant black diamond hill is the first one attempted by each avid snow skier and in (12b) the relevant moment is the one that each father dreads when his oldest child leaves home.

We have previously argued (Doran \& Ward 2015b) that the distributed interpretation of the demonstrative may be available even in the absence a quantified expression, as in (13): 
(13) Here are 3 steps to help getting you to take that first plunge underwater as a certified Scuba Diver. [corpus]

Here, the value of the demonstrative co-varies with whoever it is that is reading the web-page, rather than being interpreted under the scope of a quantified expression. Hence, the distributed interpretation is not wholly explained by the presence of a quantified expression.

Our analysis provides a straightforward account of these examples. Note that in the examples with a distributed interpretation, a culturally-shared scenario is available to license the relevant interpretation, e.g. a skier's first black diamond run (12a), the first child to leave home (12b), or a scuba diver's first dive (13). Our analysis predicts that the demonstratives in these examples will be felicitous, provided that the speaker has a basis for presuming that the hearer will be familiar with the relevant scenarios. Thus, we can provide a unified account of the distributed interpretation: one that involves culturally-familiar scenarios and community copresence.

Finally, our analysis can be straightforwardly extended to explain a popular meme in social media in which the demonstrative NP is presented as a complete utterance. Consider (14):

$$
\begin{aligned}
& \text { a. That moment when you step on a LEGO. [corpus] } \\
& \text { b. That moment when someone sneezes on you. [corpus] }
\end{aligned}
$$

In each of these examples, the demonstrative evokes a scenario; in (14a) the 'step-on-a-pointytoy' scenario is presumably familiar to an audience of parents and the demonstrative is felicitous when the speaker assumes that the hearer will be able to identify that scenario. However, in (14b), the speaker is treating the 'getting-sneezed-on' scenario as familiar in order to implicate that the audience can relate to such situations, without it actually constituting a culturallyfamiliar scenario. That is, while there is no culturally shared scenario of getting sneezed on, the use of the demonstrative here signals that the speaker is treating the relevant scenario as if it were a familiar scenario. In doing so, the use of the demonstrative in this meme allows the speaker to introduce a potentially novel situation but to treat it as if it were shared knowledge.

In this paper, we have shown that, as with other non-deictic uses of demonstratives, this use of the demonstrative is subject to a familiarity condition. However, what is distinct about this use of the distal demonstrative is that it is licensed by familiarity with culturally-familiar scenarios, which are assumed to be familiar to members of particular social groups. Thus, we see that the familiarity condition on demonstratives is realized differently across the different uses, depending upon particular features of the discourse context.

\section{References}

Clark, H. H. and C. R. Marshall. 1981. Definite reference and mutual knowledge. In A.K. Joshi, B. L. Webber, and I.A. Sag (eds.), Elements of Discourse Understanding. Cambridge: Cambridge University Press, 10-63.

Doran, R. and G. Ward. 2015a. Proximal demonstratives in predicate NPs. BLS 41, 61-70.

Doran, R. and G. Ward. 2015b. Demonstratives with distributed interpretations. CLS 51, 129136.

Diessel, H. 1999. Demonstratives: Form, Function, and Grammaticalization. Amsterdam/Philadelphia: Benjamins. 
Elbourne, P. 2008. Demonstratives as individual concepts. Linguistics and Philosophy 31(4): 409-466.

Fillmore, C. 1982. Frame semantics. In Linguistics in the Morning Calm. Seoul, Hanshin Publishing Co., 111-137.

Gundel, J.K., N. Hedberg, and R. Zacharski. 1993. Cognitive status and the form of referring expressions in discourse. Language 69:274-307.

Joshi, A.K. 1982. Mutual beliefs in question-answer systems. In N. Smith (ed.), Mutual Knowledge. New York: Academic Press, 181-197.

Kaplan, D. 1989. Demonstratives. In J. Almog, J. Perry and H. Wettstein, (eds.), Themes from Kaplan. Oxford: Oxford University Press, 481-563.

King, J. C. 2001. Complex Demonstratives. Cambridge, MA: MIT Press.

Levinson, S. 2004. Deixis. In L. R. Horn and G. Ward (eds.), Handbook of Pragmatics. Oxford: Blackwell, 97-121. 